\title{
Staphylococci with markers of antibiotic resistance collected from blood cultures
}

\author{
Vittorio Focarelli, Vincenzo Rondinelli, Stefania Giglio, Raffaele Saraceno, Pasquale \\ Minchella, Maria Gabriella Lepore, Nicola liritano, Paolo Opipari, Sandra Castagna,Teresa \\ Alcaro, Rosanna Masciari \\ Virologia e Microbiologia, Azienda Ospedaliera Pugliese-Ciaccio, Presidio Pugliese, Catanzaro
}

Key words: Blood culture, Beta-lactamase, Methicillin resistance, Antibiotic therapy

\section{Emocolture da Stafilococchi con markers di resistenza}

\section{SUMMARY}

Introduction: Blood culture is still the gold standard for the detection of the causative agent of sepsis. Especially in intensive care patients and those with vascular catheters, the most common organisms isolated are coagulase-negative staphylococci (CoNS) and Staphylococcus aureus, both characterized by multidrug resistance. Purposes of our work are the study of the incidence of markers of resistance in staphylococci and evaluation of potential changes over the years.

Materials and methods: In the period January 2008-June 20II 5239 blood cultures were analyzed. They were mainly obtained from the departments of Intensive Care, Cardiology, Hematology, General Medicine, Emergency Medicine, Infectious Diseases, Oncology, Pulmonology and Pediatric Hematoncology. The vials containing the blood were incubated in the BACTEC 9/20 automated tool of Becton Dickinson and susceptibility testing performed with the Phoenix instrument of the same company.

Results: Within a total of 5239 blood cultures, 3967 (75.7\%) were negative and 1272 (24.3\%) positive.

Fungi were isolated in $6.2 \%$ (79) of the positive ones, Gram-negative bacteria in 24.6\% (313) and Gram-positive bacteria in $69.2 \%(880)$. Within the latter, $187(21.2 \%)$ were not staphylococcal isolates, $693(78.8 \%)$ were stafiloccocci mainly represented by S. epidermidis, S. aureus, S. hominis, S. haemolyticus and S. saprophyticus. Of the 693 staphylococcal isolates, $436(62.9 \%)$ were b lactamase producers, and between them $336(77.1 \%)$ were methicillin resistant, while only 3 of $436(0.69 \%)$ were $S$. aureus resistant to vancomycin as well. The incidence of markers of resistance was very high, especially in

patients in intensive care and cardiac surgery, who are usually subjected to combined antibiotic therapy. In the three years studied there were no statistically significant differences in the resistance of staphylococci.

Conclusions: The data show an alarming high number of multi-resistant staphylococci, which is often a real therapeutic challenge for the clinician.

The interpretation of the meaning of the isolation of CoNS entails a thorough evaluation because they are commonly isolated from contaminated blood cultures.

\section{INTRODUZIONE}

L'emocoltura rappresenta tuttora il gold standard (5) nella rilevazione dell'agente eziologico della sepsi. I più comuni microrganismi isolati, specie nei pazienti in terapia intensiva e portatori di cateteri vascolari, sono gli Stafilococchi coagulasinegativi (CoNS) e lo Staphylococcus aureus, entrambi caratterizzati da multi resistenza $(2,4)$. Scopi del nostro lavoro sono lo studio dell'incidenza dei marker di resistenza negli stafilococchi e la valutazione di eventuali modifiche nel corso degli anni.

\section{MATERIALI E METODI}

Nel periodo gennaio 2008-giugno 2011 abbiamo analizzato 5239 emocolture provenienti principal- mente dai reparti di Terapia Intensiva, Cardiochirurgia, Ematologia, Medicina Generale, Medicina d'Urgenza, Malattie Infettive, Oncologia, Pneumologia ed Ematoncologia Pediatrica. I flaconi contenenti il sangue sono stati incubati nello strumento automatico Bactec 9120 della Becton Dickinson ed i test di sensibilità effettuati con lo strumento Phoenix della stessa ditta.

I ceppi batterici di riferimento (ditta Oxoid) utilizzati sono stati ATCC 29213 per Staphylococcus aureus, ATCC 43300 per Staphylococcus aureus (MRSA), ATCC 14990 per Staphylococcus epidermidis, ATCC 27844 per Staphylococcus hominis, ATCC 49907 per Staphylococcus saprophyticus.

\section{Corresponding author: Rosanna Masciari}

Virologia e Microbiologia - Azienda Ospedaliera Pugliese-Ciaccio

Via Luigi Pascali, 30 - 88I00 Catanzaro - Tel.: 096I 725069 - Fax:096I 883490

E-mail:r.masciari@teletu.it 


\section{RISULTATI}

Delle 5239 emocolture 3967 sono risultate negative $(75.7 \%)$ e 1272 positive (24.3\%) (Figura I). Nel $6.2 \%$ (79) delle positive sono stati isolati miceti, nel 24.6\% (313) batteri Gram negativi e nel $69.2 \%$ (880) batteri Gram positivi (Tabella 1). Di quest'ultimi, $187(21.2 \%)$ sono isolati non stafilococcici; 693 (78.8\%) sono invece stafilococchi rappresentati prevalentemente da $S$. epidermidis, S. aureus, S. hominis, S. haemolyticus e $S$. saprophyticus. Dei 693 stafilococchi isolati, 436 (62.9\%) sono produttori di $\beta$ lattamasi, e tra questi ben $336(77.1 \%)$ sono anche meticillino resistenti, mentre solo 3 su $436(0.69 \%)$ sono $S$. aureus anche vancomicino resistenti. L'incidenza dei marker di resistenza è stata molto elevata soprattutto nei pazienti della Rianimazione e della Cardiochirurgia, tutti in genere sottoposti a terapie antibiotiche combinate. Nei tre anni oggetto di studio non sono state rilevate differenze statisticamente significative nell'ambito delle resistenze degli stafilococchi.

\section{CONCLUSIONI}

I nostri dati ci permettono di fare, per prima cosa, un'importante constatazione di ordine generale: l'emocoltura è un esame di laboratorio che viene

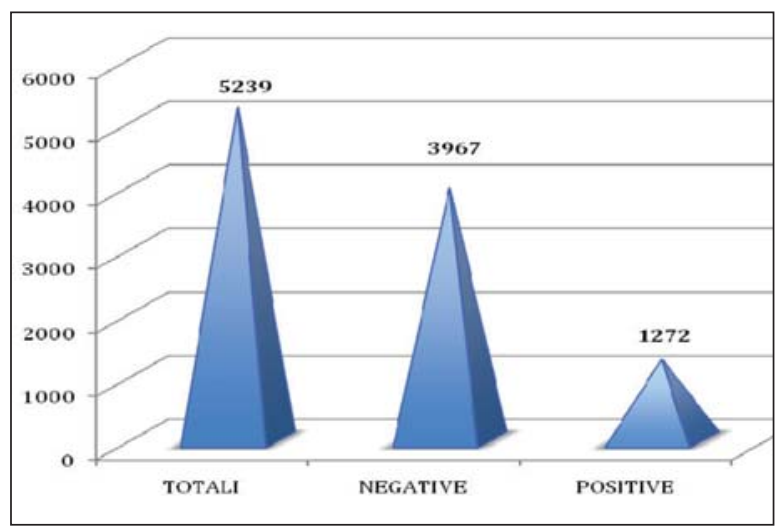

Figura I. Emocolture (5239) da gennaio 2008 a giugno 2011 . richiesto in quantità ridotta in rapporto alla reale importanza diagnostica. I campioni a noi pervenuti nell'arco di 42 mesi non sono certo numerosi. Tale evenienza potrebbe trovare una motivazione nel fatto che il risultato dell'emocoltura dipende da tantissimi fattori (il volume del campione, il momento del prelievo, la sua accuratezza, la terapia antibiotica in corso, la contaminazione del campione, il numero di prelievi per evento settico, la sede di prelievo, le difficoltà interpretative, ecc.) che spesso sono considerati da molti clinici così limitanti da metterne in discussione l'effettivo valore.

I dati evidenziano comunque una preoccupante multiresistenza degli stafilococchi (3), che spesso costituisce una vera e propria sfida terapeutica.

L'interpretazione del significato dell'isolamento di CoNS comporta un'approfondita valutazione poiché essi sono comunemente isolati da emocolture contaminate (1).

Tabella I. Risultati emocolture positive

\begin{tabular}{lc}
\hline EMOCOLTURE POSITIVE ( I 272) & N (\%) \\
\hline Miceti & $79(6.2 \%)$ \\
\hline Batteri Gram negativi & $313(24.6 \%)$ \\
\hline Batteri Gram positivi & $880(69.2 \%)$ \\
\hline
\end{tabular}

\section{BIBLIOGRAFIA}

1. Beekmann SE, Diekema DJ, Doern GV. Determining the clinical significance of coagulase-negative staphylococci isolated from blood cultures. Infect Control Hosp Epidemiol 2005; 26: 559-66.

2. Boucher HW, Corey GR. Epidemiology of methicillin-resistant Staphylococcus aureus. Clin Infect Dis, 2008; 46: 344-9.

3. Courvalin P. Predictable and unpredictable evolution of antibiotic resistance. J Intern Med., 2008; 264: 416.

4. Livermore DM. Antibiotic resistance in staphylococci. Int J Antimicrob Agents. 2000; 16: 3-10.

5. Peters RP, van Agtmael MA, Danner SA, Savelkoul PH, Vandenbroucke-Grauls CM. New developments in the diagnosis of bloodstream infections. The Lancet Infectious Diseases, 2004; 4: 751-60. 